\title{
Spiritualität in der Cura sui, im interdisziplinären Team und in der Arzt-Patienten-Beziehung
}

\section{Helen Hochreutener}

MAS in Theology of Spirituality, Kursleiterin Lassalle-Haus Bad Schönbrunn

Die Autorin hat eine Masterarbeit verfasst zum Thema: «Medizin und Spiritualität. Spiritual Care als theologischmedizinische Herausforderung. Aspekte um Verhältnis von Medizin und Spiritualität, zu Heil und Heilung in der christlichen Tradition sowie zu Ansätzen von Spiritual Care heute». Theologische Fakultät der Universität Freiburg; 2014.
Korrespondenz:

Dr. med. Helen Hochreutener Bahnhofstrasse 29

CH-3800 Interlaken

Tel. 0338232333

Fax 0338232334

helene.hochreutener[at]hin.ch

\section{Spiritualität als Ressource in der heutigen medizinischen Forschung und Lehre}

Mit wissenschaftlichen Methoden wird seit einigen Jahren erforscht, was die spirituelle Betreuung, Spiritual Care, in der gemeinsamen Sorge für den Kranken in all seinen Dimensionen als interdisziplinäre Aufgabe bringt. Forscher gehen der Frage nach, wie Patienten und Patientinnen sensibel und kompetent ganzheitlich begleitet und betreut werden können, welche Anliegen und Bedürfnisse sie haben und welche konkreten Kompetenzen notwendig sind, um die dahinterliegenden Ressourcen zu erkennen und in den Behandlungs- und Betreuungsplan zu integrieren [1-6].

\section{Kriterien für eine verantwortete, tragfähige und lebensdienliche Spiritualität}

Systematische, sinnvolle Kriterien können zu einer verantworteten, tragfähigen und lebensdienlichen Spiritualität führen.

Für das Konzept «Spiritual Care» wird hier nur kurz auf einfache und praktische Kriterien einer guten Spiritualität hingewiesen: Ist die Lebenspraxis lebensbehindernd oder lebensfördernd, vertröstend oder ermutigend, stabilisierend oder befreiend? aber doch eine Orientierung geben für die Beurteilung der Güte einer gelebten Spiritualität [7].

\section{Spiritual Care und die Selbstsorge, das inter- disziplinäre Team, die Arzt-Patienten-Beziehung} Das Konzept Spiritual Care bedeutet die umfassende Sorge für den Menschen. Sie nimmt den Menschen ganzheitlich in seiner bio-psycho-sozio-spirituellen Dimension wahr.

\section{Selbstsorge (Cura sui)}

In der Selbstsorge erhält der Mensch sich in Eigenverantwortung gesund. So kann er für sein Leben, seine Beziehungen und seinen Beruf Orientierung und Sinn finden und sich selbst spirituell-reflektiert verorten. Ein tragendes Fundament kann durch widrige Lebensumstände Halt geben und die Resilienz stärken. Darüber hinaus schenken Vertrauen und Hoffnung gelingende zwischenmenschliche Beziehungen und eine gesunde Selbstliebe. Eine gelebte spirituelle oder religiöse Praxis kann eine gute Ressource sein. Je nach biographisch-existentiellen Erfahrungen kann jedoch auch eine averse Reaktion gegen alles Religiöse auftreten, die dann als solche erkannt werden muss, da sie kontraproduktiv ist.

\section{«Der Arzt kann an einem geeigneten Zeitpunkt dem Patienten signalisieren, dass er offen ist für die spirituelle Dimension.»}

Wird das gegenwärtige Leben wertgeschätzt? Wird ein ganzheitliches gelingendes Leben gefördert? Wird etwas Innerweltliches verabsolutiert? Werden etwa Symbole mit der Wirklichkeit verwechselt? Werden mythische Bilder verobjektiviert?

Die gelebte Spiritualität soll alltagstauglich sein, den Körper, die Sinne und die Affekte einbeziehen und eine klare und zugängliche Sprache aufweisen. Kriterien dafür sind: gleiche Würde für alle, Zugang zur Gemeinschaft für alle, Offenheit für andere Spiritualitäten, Kulturen und Traditionen, geteilte Macht («Empowerment» für alle), Wachheit für sozioökonomische, ökologische und politische Probleme, Empathie für Menschen im Dunkeln, Zulassen von kritischen Fragen. Diese Indizien für eine tragfähige Spiritualität sind zwar nicht vollständig, können
Jeder Mensch lernt am besten durch Selbsterfahrung, was guttut und was schadet. Nach Lebenskrisen entwickelt er meist ein besonders gutes Gespür dafür. In einer grossen, repräsentativen, semiquantitativen Studie haben Fegg und Mitarbeiter in Deutschland sinnstiftende Bereiche im Leben untersucht: Als wichtige Bereiche haben sich familiäre und freundschaftliche Beziehungen, Freizeit, Religiosität und Natur herausgestellt. Bei gesunden, jüngeren Menschen sind Arbeit und Studium sehr wichtig, bei kranken, älteren Menschen hingegen Gesundheit, Tiere und Natur [8].

Aus der Geschichte der christlichen Spiritualität sind Wege und Methoden bekannt, die im Rahmen von Spiritual Care geeignet sind, der Selbstsorge zu dienen. Exemplarisch soll hier kurz auf die Spirituali- 


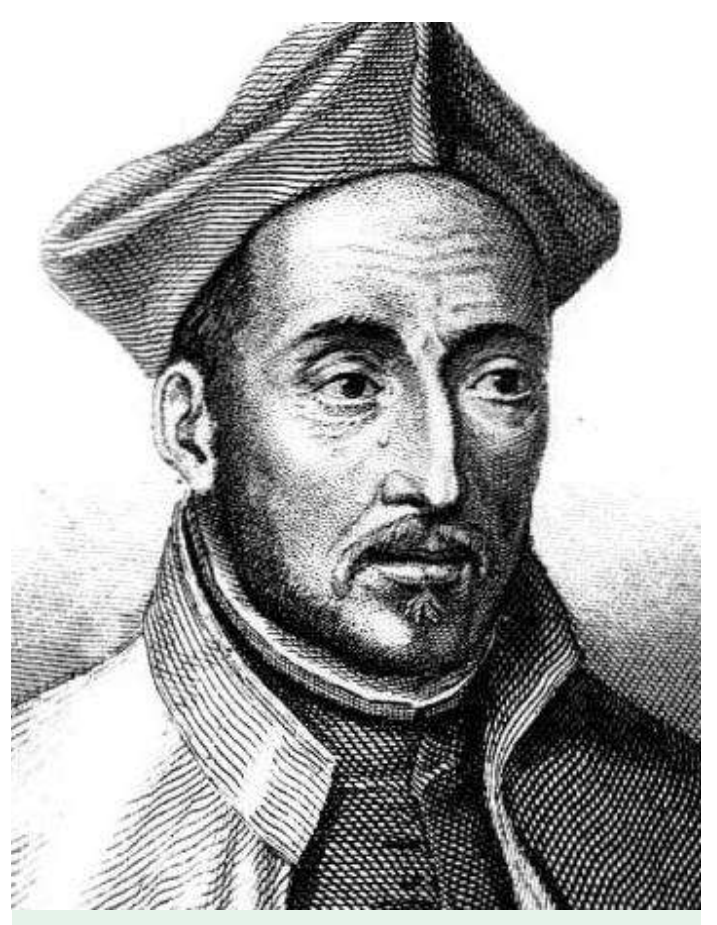

Leitete Menschen an, intellektuell zu reifen und stimmige (Lebens-)Entscheidungen zu treffen: Ignatius von Loyola (1491-1556). Sein Konzept liefert noch heute wertvolle Anregungen für Spiritual Care.

tät der Wüstenväter und des Ignatius von Loyola eingegangen werden, da sie besonders geeignet sind, auf die Bedürfnisse des heutigen Menschen und der heutigen Medizin zu antworten.

Evagrius Ponticus hat die Weisheit der Wüstenväter systematisiert, die als ursprüngliche Form vieler Aspekte der heutigen Psychologie und Psychotherapie gelesen werden kann. Beinahe alle psychischen und psychosomatischen Störungen waren im Wesentlichen damals schon bekannt. So wurden vitale, affektive und geistige Grundbedürfnisse beschrieben, deren akzentuierte Form als verzerrte Leidenschaften (pathe) erkannt wurden. Bestimmte Verhaltensweisen zu deren Kontrolle oder Heilung wurden ebenso empfohlen. Die Akedia (Überdruss, Gleichgültigkeit) wurde als Krise in der Lebensmitte beschrieben, die der Psychiater Daniel Hell als Vorläufer der heutigen Midlife-Crisis deutet. Der damalige empfohlene spirituelle Weg diente dazu, die Apatheia zu erreichen (griechisch $\alpha$ ma $\alpha \varepsilon \varepsilon$, , Gelassenheit, innere Freiheit) [9].

Die Exerzitien nach Ignatius sind ein weiterer agogisch gut konzipierter spiritueller Weg zum Erreichen der inneren Freiheit (ignatianisch «Indifferenz»). So kann der Mensch menschlich und intellektuell reifen und stimmige (Lebens-)Entscheidungen treffen. Der Übende wird genügend frei von ungeordneten Anhänglichkeiten (Abhängigkeiten) und damit frei für bewusst gewählte Ziele [10].

\section{Spiritual Care im interdisziplinären Team}

Eine spirituelle Führungskompetenz kann zu einem gelingenden kommunikativen Umgang im interdisziplinären Team beitragen, so dass ein gutes, tragendes Klima unter allen Mitarbeitern im Gesundheitswesen entsteht und das Klima nicht durch Mobbing oder andere Friktionen vergiftet wird.

Zwei Methoden aus der christlichen Spiritualitätsgeschichte sollen hier kurz erwähnt werden, in die zusätzlich auch moderne Erkenntnisse der Humanwissenschaften einfliessen.

Aufbauend auf der ignatianischen Spiritualität wird ein Ansatz zu gemeinsamen, tragenden Entscheidungen vorgestellt. Der günstige Augenblick, eine Entscheidung zu treffen, ist wichtig. Wählen und entscheiden zu können, gehören zu den typischen Merkmalen des freien Menschen. Der Kairos (griechisch кaıрos) ist der intuitiv gespürte rechte Augenblick, während der Ausdruck Chronos (griechisch xpovoऽ) für die Zeit steht, die objektiv-linear abläuft. Gelegentlich werden dringliche Entscheidungen unter Druck gefasst. Ignatius von Loyola leitet mit speziellen Methoden dazu an, sich für wesentliche Entscheidungen «indifferent» zu machen. Noch weiter bestehende ungeordnete Regungen sollen wahrgenommen werden, jedoch die Entscheidungen nicht bestimmen.

Nach Ignatius gibt es drei Weisen, sich zu entscheiden: 1. die unmittelbare Intuition, 2. das Wahrnehmen der inneren Regungen (Affekte) und 3. das argumentativ-rationale Abwägen der Pros und Kontras.

Auf dem Hintergrund der «Deliberatio Patrum» hat Bernhard Waldmüller einen Leitfaden des spirituellen Umgangs mit gemeinsamen Entscheidungen vorgelegt, der sich für Entscheidungen im interdisziplinären Team gut anwenden lässt [11].

Ärzte tun gut daran, auch das Thema Macht zu reflektieren. Wer Macht ausübt, kann das Leben für sich und andere in vielfältiger Weise bestimmen. Macht ist grundsätzlich gut, da der Mensch kreativ sein Umfeld mitgestalten kann. Macht ist Mittel zum Ziel, soll also nicht Selbstzweck sein. Nach Ignatius sollen die Mächtigen ihre Macht für gute Zwecke einsetzen. Ein Amt oder eine Aufgabe gut zu führen, bedeutet, nicht aus Eigeninteresse einen Entscheid zu fällen, sondern eine Win-win-Situation für alle Beteiligten zu schaffen $[12,13]$.

\section{Spiritual Care in der Arzt-Patienten-Beziehung}

Die christliche Anthropologie stellt den Menschen als Person in den Mittelpunkt. Eine «Person-Zentrierung» wirkt sich verändernd auf das leitende medizinische Behandlungskonzept aus. Die Arzt-PatientenKommunikation gewinnt mehr an Bedeutung. In der narrativen Medizin wird der Patient ermutigt, von seiner Krankheitserfahrung zu erzählen und selbst zu deuten. Auf diese Weise kommt der Patient als unverwechselbare Person in den Blick. 
Krankheit ist auf der körperlichen Ebene oft mit Schmerzen und Einschränkungen verbunden, auf der sozialen Ebene mit Isolation und Einsamkeit und auf der psychischen Ebene mit Angst und Verzweiflung.

In der Lebensqualitätsforschung wird neben den objektiven Lebensbedingungen immer stärker auch das subjektive Wohlbefinden wie Zufriedenheit und Glücklich-Sein als wichtiges Kriterium betrachtet. Zur Prävention und zur Bewältigung von körperlichen Erkrankungen tragen persönliche Ressourcen, z.B. Lebenszufriedenheit, Sinnerfüllung sowie säkular und religiös motivierte Bewältigungsformen (coping strategies) bei. Heilen heisst also nicht nur Beseitigen von Krankheit, sondern Ermöglichen eines als subjektiv sinnvoll erfahrenen Lebens. Dazu soll die Arzt-Patienten-Beziehung geprägt sein durch Empathie, Wohlwollen und Mitmenschlichkeit. Die Sensibilität für die spirituelle Dimension in dieser Beziehung kann zu einem achtsameren, ganzheitlicheren Umgang mit dem Patienten führen.

Der Arzt kann an einem geeigneten Zeitpunkt in der ärztlichen Betreuung dem Patienten signalisieren, dass er offen ist für die spirituelle Dimension, indem er beim Erheben der Anamnese den Patienten auf seine spirituell-religiösen Bedürfnisse und Ressourcen anspricht. Standardisierte, semiquantitative Anamnese-Interviews dazu wurden mittlerweile erarbeitet und evaluiert (SPIR-Anamnese-Interview, [2]). Je nach Bedarf lässt es sich situationsbezogen erweitern. Sollte der Patient im Augenblick kein solches Gespräch führen wollen, ist sein Wille Gebot. Er hat jedenfalls den Hinweis bekommen, dass er, falls gewünscht, das Gespräch fortsetzen oder sich anderweitig diesbezüglich in Verbindung setzen kann.

Eine schwere Krankheit zwingt den Menschen oft dazu, sich mit dem Sinn der Krankheit, dem Sinn des eigenen Lebens und über die eigene Begrenztheit und den Tod nachzudenken. Solche Fragen sind allgemein menschliche Fragen und nicht an eine spezifische Religion oder Spiritualität gebunden. Wenn der Mensch sich auf diese Fragen einlässt, wird die existentielle Erfahrung einen spirituellen Reifungsprozess auslösen. Oft zwingt auch eine Krankheit, bisherige eigene Lebenswünsche loszulassen, Unfertiges anzunehmen und sich mit dem Leben zu versöhnen. Oft wächst der Mensch über sich hinaus, transzendiert sich also selbst und findet seinen Lebenssinn im Sein-für-Andere oder Sein-für-etwas-Anderes. Im Gewahr-Werden der eigenen Grenzen, in der Lebenskrise kann der Mensch das entscheidend Wesentliche im eigenen Leben finden. Die Leidfrage ist die zentrale Bewährungsprobe für eine Spiritualität, soll sie doch zeigen, ob sie Ressourcen für eine subjektive Bewältigung von Leid und Tod zur Verfügung zu stellen vermag. Der Begleitende soll keine falschen Hoffnungen geben, jedoch auch nicht jede Hoffnung auf Heilung nehmen, sondern Orientierung schenken. Leid, Schmerz und Tod gehören konstitutiv zur Existenz des Menschen. Der bewusst Sterbende kann zum Schluss kommen, dass der Sinn des Lebens in etwas
Umfassenderem liegen muss. Im Umgang mit der Schuldfrage muss oft in einer spirituell-existentiellen Reifung unerklärliches Leid stehengelassen werden, um nicht in kurzschlüssige Erklärungen zu fallen. Die pastorale Praxis soll sich dabei nach dem Bedürfnis der Patienten richten [14].

Die Palliativmedizin und die Palliative Care haben sich prominent in ihrem Leitbild der ganzheitlichen, inklusive der spirituellen Verantwortung verpflichtet [2, 8]. Der Palliativmediziner Gian Domenico Borasio erwähnt für die Sterbebegleitung folgende wesentliche Bedürfnisse des Patienten und Sterbenden: Kommunikation, eine optimale palliativ-medizinische Therapie, psychosoziale Betreuung und spirituelle Begleitung [15].

\section{Literatur}

1 Koenig H, King D, Benner Carson V.

Handbook of Religion and Health. Second Edition.

Oxford: University Press; 2012.

2 Frick E, Roser T (Hrsg.). Spiritualität und Medizin. Gemeinsame Sorge für den kranken Menschen. Münchner Reihe Palliative Care. Palliativmedizin Palliativpflege - Hospizarbeit. Stuttgart: Kohlhammer; 2009. www.spiritualocare.de

3 www.uniweiterbildung.ch/studienangebot/ kursdetails/?tx_x4econgress_ pi1\%5BshowUid\%5D=779

4 www.lassalle-haus.org Fortbildungsveranstaltungen Medizin und Spiritualität

5 Internationale Gesellschaft für Gesundheit und Spiritualität (Hrsg.) unter Leitung von Frick E, Traugott R. Spiritual Care. Zeitschrift für Spiritualität in den Gesundheitsberufen. Stuttgart: Kohlhammer. www.spiritual-care-online.de

6 www.ecrsh.eu/

Organisator Dr. med. René Hefti Langenthal.

7 Hiestand FX, Müller C. Indizien einer tragfähigen Spiritualität. In: Leutwyler Samuel, Nägeli M (Hrsg.). Spiritualität und Wissenschaft. Zürich: vdf Hochschulverlag AG ETH; 2005. S 269-83.

8 Fegg M, Kramer M et al. Meaning in Life in the Federal Republic of Germany: results of a representative survey with the Schedule for Meaning in Life Evaluation (SMiLE). Journal of Pain and Symptom Management. 2007;35(4):356-64.

9 Evagrios P. Der Praktikos (Der Mönch). Weisungen der Väter. Bunge Gabriel (Hrsg. und Übersetzer). Band 6. Verbesserte Auflage. Beuroner Kunstverlag; 2008.

10 Ignatius von Loyola. Die Exerzitien. Christliche Meister. Einsiedeln: Johannes Verlag; 2005.

11 Waldmüller B. Gemeinsam entscheiden. Ignatianische Impulse. Würzburg: Echter Verlag; 2008.

12 Kiechle S. Macht ausüben. Ignatianische Impulse. Würzburg: Echter Verlag; 2005.

13 Kiechle S. Sich entscheiden. Ignatianische Impulse. Würzburg: Echter Verlag; 2008.

14 Schaupp W. Spirituelle Dimensionen des Krankseins. In: Körtner U, Müller S, Kletcka-Pulker M, Inthorn J (Hrsg.). Spiritualität, Religion und Kultur am Krankenbett. Schriftenreihe Ethik und Recht in der Medizin. Wien/New York: Springer Verlag; 2009. S. $165-75$.

15 Borasio GD. Über das Sterben. München: Verlag C. H. Beck; 2012. 